\title{
Androgen receptor axis-targeted agents
}

\author{
Anil Kapoor, MD, FRCSC;i Sebastien J. Hotte, MD, FRCPC ${ }^{2}$
}

'Professor of Surgery (Urology), McMaster University, Associate Editor (Oncology), Canadian Urological Association Journal (CUA), Hamilton, ON, Canada; ${ }^{2}$ Associate Professor, Department of Oncology, Division of Medical Oncology, McMaster University; Medical Oncologist, Juravinski Cancer Centre, Hamilton, ON, Canada

Cite as: Can Urol Assoc J 2016;10(7-8-Suppl3):S146-8. http://dx.doi.org/10.5489/cuaj.4057

Biologic heterogeneity may affect response to androgen receptortargeting therapies

When used in combination with medical castration, abiraterone acetate augments cytoreduction in patients with localized high-risk prostate cancer. Preliminary results in the castrate-resistant population showed that the combination of abiraterone acetate and enzalutamide was able to block the adaptive increase in serum testosterone seen with enzalutamide, with no unanticipated toxicities or pharmacokinetic interactions. ${ }^{1}$ At ASCO 2016, these same researchers presented results of their trial exploring whether the addition of enzalutamide to combination treatment with abiraterone acetate and a luteinizing hormone-releasing hormone (LHRH) analogue would achieve greater cytoreduction in castration-naïve localized prostate cancer - an important unmet therapeutic need. ${ }^{2}$ Patients with localized high-risk prostate cancer (clinical stage T1c/T2 and Gleason score 8 or higher, or stage T2b or higher and Gleason score 7 or higher and prostate-specific antigen [PSA] higher than 10 $\mathrm{ng} / \mathrm{mL}$ ) were randomly assigned in a 2:1 ratio to 24 weeks of treatment with either abiraterone plus enzalutamide plus LHRH analogue or abiraterone acetate plus LHRH analogue.

Despite nearly undetectable PSA levels in both groups of patients, there was a trend toward more tumour downstaging to pathology stage ypT2N0 or lower in the group that received abiraterone acetate plus LHRH analogue than in the group that received enzalutamide plus abiraterone acetate plus LHRH analogue. The presence of ARV7 was significantly more frequent in tumours treated with the three-drug combination including enzalutamide $(p<0.05)$. Pathological stage ypT2N0 or lower was associated with lower Ki67 $(p<0.005)$ and increased expression of canonical androgen receptor (AR) signaling components AR-N, ARC19, CYP17 $(p<0.005)$, but not PSA $(p<0.17)$. Taken together, these findings suggest that biologic heterogeneity warrant consideration when applying AR-targeting therapies in men with hormone-naïve, high-risk prostate cancer.

PSA declines with enzalutamide treatment are associated with clinical benefit and improved overall survival in men with metastatic castration-resistant prostate cancer

The AFFIRM trial showed a significant increase in overall survival (OS) with enzalutamide vs. placebo in men with metastatic castrate-resistant prostate cancer (mCRPC) following chemotherapy, as well as a significant decline in PSA levels. A post-hoc analysis of the AFFIRM results was conducted to explore the potential for PSA decline to function as a surrogate for OS in this patient population. ${ }^{3}$ Men enrolled in the AFFIRM trial were grouped according to their maximal unconfirmed PSA decline during the first 90 days of treatment, and then PSA decline was assessed for its association with OS, progression-free survival (PFS), and pain response. Enzalutamide was associated with greater OS (hazard ratio $[H R] 0.63 ; p<0.001)$ and higher rates of unconfirmed PSA declines compared with placebo (odds ratio $[\mathrm{OR}]>19.0$; $p<0.001)$. Greater declines in PSA were associated with longer OS, PSA PFS, radiographic PFS, and higher pain response when compared with PSA increase/no decline. PSA declines of $30 \%$ or greater or $50 \%$ or greater within the first 90 days of treatment were associated with a proportion of treatment effect explained in the range of 1.07-1.29 (95\% confidence interval $[\mathrm{Cl}]$ lower bounds $\geq 0.63$ and $\geq 0.55$ for unconfirmed and confirmed PSA decline, respectively), in which treatment was no longer significant after adjustment for the decline measures $(p>0.20)$. Because few PSA declines were seen with placebo, full surrogate equivalency of PSA decline on OS could not be established; however, PSA decline explained a substantial amount of the effect of enzalutamide on survival. These results suggest that a lack of PSA declines may be used to identify men with mCRPC who develop early resistance to AR-inhibitor treatment, helping to guide treatment for these men. 
Adjuvant enzalutamide in men with high-risk prostate cancer after radical prostatectomy yields low rate of biochemical recurrence

High-risk prostate cancer remains a clinical challenge. A large proportion of affected men develop biochemical recurrence or metastases within two years; currently, the only adjuvant therapy that has demonstrated efficacy is radiation therapy for selected men with high-risk features who have undergone radical prostatectomy. With the advent of novel AR inhibitors as the standard of care in CRPC, these agents are currently being explored for their potential to impact outcomes in men with high-risk prostate cancer following radical prostatectomy. Ornstein and colleagues presented results of a phase 2 study evaluating the efficacy/safety of enzalutamide in 42 men with high-risk prostate cancer who had undetectable PSA within three months of undergoing radical prostatectomy. ${ }^{4}$ Patients were treated with enzalutamide $160 \mathrm{mg} /$ day in a 28-day cycle until disease progression, drug intolerability, consent withdrawal, or completion of the study at 24 months. After a median followup of 16.9 months, 40 of 41 patients with followup information remained free of biochemical recurrence. Fourteen men had discontinued treatment - eight because they had completed the study treatment, three due to Grade 3 adverse events, one due to non-enzalutamide-related medical issues after 15 cycles, one due to biochemical recurrence after 15 cycles, and one because the patient withdrew consent after 21 cycles. Dose reductions were required in two patients due to myalgia/arthralgia. Neurological adverse events were similar to those observed in CRPC trials. Despite the heterogeneity of this population, the lack of a comparator arm, and the short followup period, the small rate of biochemical recurrence is encouraging, supporting further exploration of these agents in the adjuvant setting.

\section{Next-generation AR axis-targeted agents show anticancer activity in men who progressed to CRPC after upfront androgen-deprivation therapy and docetaxel in the GETUG-AFU 15 trial}

Three recent phase 3 trials have shown a survival benefit of the addition of docetaxel chemotherapy to androgen-deprivation therapy (ADT), compared with ADT alone in men with prostate cancer - GETUG-AFU 15, 5,6 CHAARTED, ${ }^{7}$ and STAMPEDE. ${ }^{8}$ These data have led to the adoption of this combination as the standard of care in fit men with hormone-naïve prostate cancer. A retrospective analysis of the GETUG-AFU 15 was conducted to evaluate the effects of treatments received beyond progression for CRPC. ${ }^{9}$ Of the 345 patients from GETUG-AFU 15 for whom data were available, 245 patients received at least one anticancer treatment for CRPC. When docetaxel was used for first-line CRPC treatment, biochemical PSA was seven months in the ADT arm and 4.1 months in the ADT plus docetaxel arm
- PSA declines were $34 \%$ and $17 \%$, respectively. When the next-generation AR-targeted therapies abiraterone and enzalutamide were used for first- or second-line treatment of CRPC, PSA declines were $66 \%$ in the ADT arm and $63 \%$ in patients treated with ADT and docetaxel. After the onset of CRPC, OS was 27.1 months in the ADT arm and 23.7 months in the ADT plus docetaxel arm. Grade 3 to 4 adverse events were rare in both groups.

\section{Cognitive impairment and central nervous system events with AR-targeted therapies}

The advent of the new hormonal agents abiraterone acetate and enzalutamide have improved the prognosis of men with mCRPC. However, recent concerns are emerging about the potential for central nervous system (CNS) effects, fatigue, and pain necessitating dose adjustments. While a number of published studies have described an association between ADT and cognitive decline/mood effects in men with prostate cancer, ${ }^{10}$ the effects of abiraterone and enzalutamide on cognition have been less well-described. An ongoing, randomized, phase 2 trial is evaluating the sequencing of abiraterone and enzalutamide in treatment-naïve patients with $\mathrm{mCRPC}$. A secondary objective of the trial is to evaluate cognitive function and depressive symptoms using the Montreal Cognitive Association (MoCA) testing for cognitive impairment and Patient Health Questionnaire (PHQ-9) for depression symptoms; preliminary results were reported at ASCO $2016 .{ }^{11} \mathrm{~A}$ total of 60 patients were randomly assigned to treatment with either abiraterone $(n=27)$ or enzalutamide $(n=33)$, with a minimum followup of three months; 10 were non-evaluable for MoCA and seven for PHQ9 data. With median baseline scores of 25 for MoCA and 3 for PHQ-9, the abiraterone and enzalutamide groups had similar baseline levels of impairment in in PHQ9 (33.3\% vs. 31.3\%) and MoCA scores (76.9\% vs. $74.2 \%$ ). Significantly more patients treated with enzalutamide than with abiraterone had a worsening depression severity score $(p=0.03)$ and there was also a trend toward a worse cognitive impairment score with enzalutamide $(p=0.20)$. More patients on enzalutamide than abiraterone reported worsening energy, appetite, and psychomotor symptoms on the PHQ-9 questions.

At ASCO 2016, Pilon and colleagues presented results of their retrospective analysis of patients in MarketScan databases who were treated with either abiraterone $(n=1493)$ or enzalutamide $(n=1510)$, and assessed for CNS, fatigue, pain, and dose reduction events. ${ }^{12}$ A total of 2196 patients taking abiraterone and 807 patients taking enzalutamide were identified. Baseline demographic, comorbidities, and disease characteristics were balanced using inverse probability of treatment weighting. After one year of followup, both CNS events and fatigue events were significantly higher in the enzalutamide-treated patients than in the abiraterone- 
Kapoor et al.

treated patients $(37.5 \%$ vs. $30.3 \% ; p=0.005$; and $28.6 \%$ vs $25.0 \% ; p=0.013)$. Dose reductions were also required in more enzalutamide-treated patients than abiraterone-treated patients $(20.0 \%$ vs. $13.6 \% ; \mathrm{p}<0.001)$.

While the results of these studies may be useful in helping to characterize the incidence of cognitive symptoms in men with $\mathrm{mCRPC}$ who are undergoing treatment with a novel hormonal therapy, the results should be interpreted with caution, as the analysis of these endpoints were not powered to make conclusions. These data are hypothesis-generating for future exploration and clarification.

\section{References}

1. Efstathiou E, Titus MA, Wen S, et al. Enzalutamide (ENZA) in combination with abiraterone acetate (AA) in bone metastatic castration resistant prostate cancer (mCRPC). J Clin Oncol 2014;32(suppl). Abstr 5000.

2. Efstathiou E, Davis J, Titus $M$, et al. Neoadjuvant enzalutamide (ENZA) and abiraterone acetate (AA) plus leuprolide acetate (LHRHa) vs. AA+ LHRHa in localized high-risk prostate cancer (LHRPC). J Clin Oncol 2016;34(suppl). Abstr 5002

3. Armstrong AJ, Saad F, Shore ND, et al. Surrogacy analysis of prostate-specific antigen (PSA) decline for improved overall survival (OS) with enzalutamide (ENZ) in AFFIRM. J Clin Oncol 2016;34(suppl). Abstr 5062.

4. Ornstein MC, Stephenson AJ, Elson P, et al. Adjuvant enzalutamide (Enza) for men with high-risk prostate cancer (HRPCa) after radical prostatectomy (RP). J Clin Oncol 2016;34(suppl). Abstr 5071.
5. Gravis G, Boher JM, Joly F, et al; GETUG. Androgen-deprivation therapy (ADT) plus docetaxel vs. ADT alone in metastatic non castrate prostate cancer: Impact of metastatic burden and long-term survival analysis of the randomized phase 3 GETUG-AFU15 trial. Eur Urol 2016;70:256-62. http://dx.doi. org/10.1016/i.eururo.2015.11.005

6. Gravis $\mathrm{G}$, Fizazi K, Joly F, et al. Androgen-deprivation therapy alone or with docetaxel in non-castrate metastatic prostate cancer (GETUG-AFU 15): A randomized, open-label, phase 3 trial. Lancet Oncol 2013;14:149-58. http://dx.doi.org/10.1016/S1470-2045(12)70560-0

7. Sweeney $C J$, Chen YH, Carducci $M$, et al. Chemohormonal therapy in metastatic hormone-sensitive prostate cancer. N Engl J Med 2015;373:737-46. http://dx.doi.org/10.1056/NEJMoal503747

8. James ND, Sydes MR, Clarke NW, et al; STAMPEDE investigators. Addition of docetaxel, zoledronic acid, or both to first-line long-term hormone therapy in prostate cancer (STAMPEDE): Survival results from an adaptive, multiarm, multistage, plafform-randomized, controlled trial. Lancet 2016;387:1163-77. http:// dx.doi.org/10.1016/S0140-6736(15)01037-5

9. Lavaud $P$, Gravis $G$, Legoupil $C$, et al. Efficacy and tolerance of treatments received beyond progression in men with metastatic hormone-naive prostate cancer treated with androgen deprivation therapy (ADT) with or without docetaxel in the GETUG-AFU 15 phase 3 trial. J Clin Oncol 2016;34 (suppl). Abstr 5080.

10. McGinty HL, Phillips KM, Jim HS, et al. Cognitive functioning in men receiving androgen-deprivation therapy for prostate cancer: A systematic review and meta-analysis. Support Care Cancer 2014;22:2271-80. http://dx.doi.org/10.1007/s00520-014-2285-1

11. Parimi S, Eigl BJ, Sunderland K, et al. Effects of abiraterone (ABI) and enzalutamide (ENZA) on cognitive impairment and depressive symptoms in patients (pts) with metastatic castration-resistant prostate cancer (m(RPC). J Clin Oncol 2016;34(suppl). Abstr 5059.

12. Pilon D, Behl AS, Gozalo L, et al. Assessment of central nervous system (CNS) and dose reduction events in patients treated with abiraterone acetate plus prednisone (AA+P) or enzalutamide (ENZ). J Clin Oncol 2016;34(suppl). Abstr 5078 